\title{
A contactless ultrasonic surface wave approachto characterize distributed cracking damage in concrete
}

\author{
Suyun Ham ${ }^{1,4}$, Homin Song ${ }^{1}$, Michael L. Oelze ${ }^{2}$, and John S. Popovics ${ }^{1,3}$
}

${ }^{1}$ The Department of Civil and Environmental Engineering, The University of Illinois, Urbana, IL 61801

${ }^{2}$ The Department of Electrical and Computer Engineering, The University of Illinois, Urbana, IL 61801

${ }^{3}$ Corresponding author: 205 N. Mathews St. Urbana, IL 61801; johnpop@illinois.edu

${ }^{4}$ Present address: Department of Civil Engineering, The University of Texas at Arlington, Arlington TX 76019

\begin{abstract}
We describe an approach that utilizes ultrasonic surface wave backscatter measurements to characterize the volume content of relatively small distributed defects (microcrack networks) in concrete. A simplified weak scattering model is used to demonstrate that the scattered wave field projected in the direction of the surface wave propagation is relatively insensitive to scatters that are smaller than the propagating wavelength, while the scattered field projected in the opposite direction is more sensitive to sub-wavelength scatterers. Distributed microcracks in the concrete serve as the small scatterers that interact with a propagating surface wave. Data from a finite element simulation were used to demonstrate the viability of the proposed approach, and also to optimize a testing configuration to collect data. Simulations were validated through experimental measurements of ultrasonic backscattered surface waves from test samples of concrete constructed with different concentrations of fiber filler $(0.0,0.3$ and $0.6 \%)$ to mimic increasing microcrack volume density and then samples with actual cracking induced by controlled thermal cycles. A surface wave was induced in the concrete samples by a $50 \mathrm{kHz}$ ultrasonic source operating $10 \mathrm{~mm}$ above the surface at an angle of incidence of $9^{\circ}$.Silicon-based miniature MEMS acoustic sensors located a few millimeters above the concrete surface both behind and in front of the sender were used to detect leaky ultrasonic surface waves emanating from concrete.A normalized backscattered energy parameter was calculated from the signals. Statistically significant differences in the normalized backscattered energy were observed between concrete samples with varying levels of simulated and actual cracking damage volume.
\end{abstract}

Keywords:Air-coupled, Backscatter, Incoherent, In situ characterization, MEMs, Microcracking, Non-Destructive Evaluation (NDE) 


\section{Introduction}

According to a 2013 report by the American Society of Civil Engineers [1], the infrastructure of the United Statesrequires a \$3.6 trillion investment to restore good operational conditionby 2020.A significant portion of the American infrastructure is composed of concrete. Concrete is a composite material that exhibits natural inhomogeneity across a broad range of length scales - from micrometers (natural capillary pores and cracks in the cement matrix) to centimeters (mineral aggregate dispersed phase). Most concrete deterioration problems involve internal material chemical and physical transformations[2].The most significant of these degradation mechanisms, freezing and thawing cycles, alkalisilica reactivity (ASR), sulfate ion attack, high temperature (e.g. fire) exposure, and mechanical overloading [3], give rise toa distributed network of fine cracks in concrete that normally propagate around, but in some cases through, the mineral aggregates and throughout the cement matrix.This type of distributed damage in concrete is called "microcracking." Figure 1 shows scanning electron microscope (SEM) images of concrete using backscattered electron microscopy technology. Figure 1(left) shows a nominally undamaged sample where the mineral aggregate (dark gray), cement binder (light gray) and air pore (black) phases are visible. Although the sample is nominally pristine, some fine cracks are still visiblein the image. The images on the right show the same material after being exposed to several extreme hot-cold $\left(70^{\circ} \mathrm{C}-0^{\circ} \mathrm{C}\right.$ ) temperature cycles. The thermal cycles cause the creation of additional thin (on the order of $1 \mu \mathrm{m}$ in width) microcracksthat exist in the cement matrix, along the aggregate-matrix interface, and in the mineral aggregate. Although small in width, microcrack networks adversely affect material mechanical strength, stiffness and permeability.Moreover, these networks increase ingress of aggressive substances into the material that enhance subsequent degradation mechanisms. It follows that microcracks adversely compromisethe resilience and sustainability of concretestructural systems. In order to apply effective monitoring and maintenance procedures and to better predict service life and monitor structural condition, a nondestructive testing (NDT) technology is required that provides accurate and sensitive characterization of distributed cracking damagein concrete, including fine microcracks.
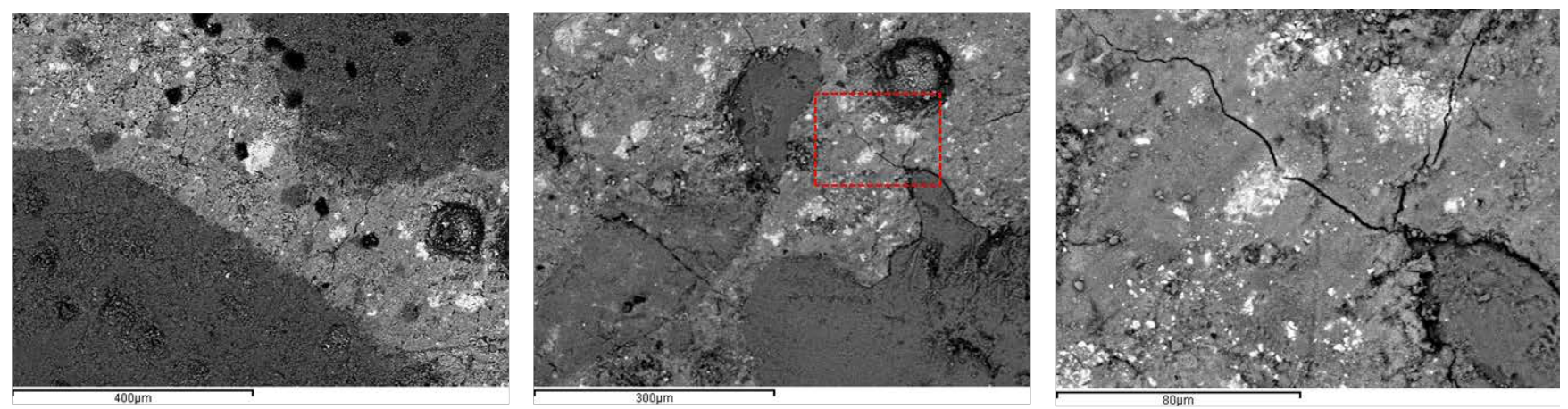

Figure 1 Scanning electron microscopy (SEM) images of concrete: (left)undamaged material, and (middle) material with microcracking damage induced by 10 thermal shock cycles; increased resolution image of region indicated by red boxshown on the right.

Most concrete infrastructure comprise large critical systems, such as bridges, pavements and buildings. Significant progress has been made in recent years toward health monitoring and NDT of large critical concrete infrastructure systemsin situ[3]. However most conventional concrete NDT methods, such as visual inspection, sounding (e.g., chain drag), pulsed microwave/radar techniques (GPR) and infrared thermography, cannot detect and characterize the extent of distributed microcracking damage in concrete [3]. Conventional mechanical wave (e.g. ultrasound) methods, which utilize 
coherent pulse propagation characteristics such as wave velocity or attenuation, have demonstratedsensitivity to large discrete cracks and defects. However these methodsare much less able to characterize the extent of microcracking damagebecause of the relatively large wavelengths with respect to the crack size needed to propagate in a highly inhomogeneous and scattering medium like concrete. To maintain coherent ultrasonic pulse character through the highly inhomogeneous material structure, relatively low frequencies (less than $100 \mathrm{kHz}$, which corresponds to approximately 4 cm wavelength for p-waves) are employed. Despite large wavelengths, the use of coherent pulse p-wave velocity [4], [5], p-wave attenuation [4]-[10]and surface wave characteristics [11] to monitor microcracking in concrete have been described, where some ability to detect and distinguish between different levels of damage extent has been reported[4], [5], [8], [10]. However, the sensitivity to low levels of cracking damage remains poor with coherent ultrasonic methods, and the results have not yet been directly related to a quantifiable measure of the damage, such as crack density.

Recent efforts to improve the sensitivity of ultrasonic measurements to microcracking damage and other microstructural modifications in concrete have focused on nonlinear [12]-[15], [16]and diffuse field measurements [17]-[23]. The nonlinear measurements use amplitude dependent softening [12]-[14] through nonlinear resonance spectroscopy or higher harmonic generation [15], [16]phenomena to extract nonlinear signal parameters. The extracted nonlinear parameters demonstrate superior sensitivity to the presence of damage, including the early stages of microcracking, as compared with conventional coherent ultrasonic wave velocity and attenuation measurements. However, some of these test configurations, for example that for nonlinear resonant spectroscopy, require test samples of specific size and geometry, and as such are laboratory-based methods that cannot be applied to large concrete structures in situ. Thus, application of nonlinear ultrasound to structures in situ remains rare.The diffuse field measurements follow a different analysis procedure, where the data are interpreted through scattered field analysis or a classical diffuse wave interpretation[20]or coda wave interferometry [17], [19].However, practical application of diffuse field methods to characterizedamage in concrete remains scarce, and as far as the authors are aware the application of ultrasonic diffuse wave measurements to concrete structures in situ have not been reported.This situation likely occurs because of limitations associated with sample size, a need for specialized high-end testing equipment, experimental limitations related to test data variability, or the ability to uniquely and reliably quantify and distinguish different microcracking damage levels. Concerning this last point, none of the research efforts with these recently developed methods report the ability to uniquely and reliably quantify and distinguish the different damage levels in concrete, i.e., by testing for statistical significance within the data. Such statistical studies are important for new measurement methods, which may be adversely affected by high test data variability.

This paper demonstrates a practical contactlessultrasonic testing approach that uses backscatter measurements to characterize distributed microcrackingdamage in concrete. Although previous research has identified that some ultrasonic and other nondestructive test data can be related to distributed damage content in concrete, the methods are either insensitive to early stages (low crack volumes) of damage, exhibit high data variability, or are not applicable to concrete structures in situ. Furthermore,many concrete NDT methods require some sort of physical contact with the concrete, which can considerably slow down the testing process and cause data inconsistency problems because of sensor coupling. Contactless sensing systems offer a solution to this problem [24], providing an effective way to characterize distributed 
cracking damage in large concrete structures. We intend to overcome the issues that hamper deployment of ultrasonic testing of concrete by employing air-coupled contactless ultrasonic surface wave tests. Here, we demonstrate that ultrasonic surface wave backscatter parameters have potential for increased sensitivity to the presence of small, relative to ultrasonic wavelength, microcracks in concretewhile at the same time providing consistent and reliable data. We are able to distinguish different levels of microcracking-type damage with a high degree of statistical confidence.Furthermore, we deploy the surface wave tests using a fully contactless air-coupled configuration, providing the potential for a practical and efficient method to evaluate in-service infrastructure elements. Finally, we suggest a physical meaning/basis for observed backscatter parameters. These contributions represent advances to the ultrasonic nondestructive testing and infrastructure healthmonitoring communities.

\section{Theory}

Mechanical wave scattering is generally represented by two categories: weak scattering and strong scattering. Weak scattering occurs when either the constitutive properties of the scatterers are close to the properties of the background material, or when the scatterersarevery small compared to the wavelength of the propagating wave. Under weak scattering conditions, simplifying approximations can be madethat lead to important conclusions relating the scattered field to the geometry, properties and size of the underlying scatterers.

To help understand the relationship between scattering direction and dependence onscatterer geometry, a Born approximation of mechanical wave scattering can be utilized. Assuming harmonic wave propagation and considering a longitudinal p-wave, $p(\vec{x}, t)=p(\vec{x}) e^{j \omega t}$, then the classical wave equation reduces to the Helmhotz equation,

$$
\nabla^{2} p(\vec{x})+k^{2} p(\vec{x})=S(\vec{x})
$$

wherep is the acoustic pressure, $k$ is the acoustic wavenumber, and $S(\vec{x})$ is the object function. Assuming plane wave propagationand invoking the Born approximation yields the scattered pressure field observed far from the scattering sourcesin volume $V_{0}[25]$,

$$
p_{s c}(\vec{r}) \approx \frac{P_{0} k^{2} e^{j k r}}{4 \pi r^{2}} \int_{V_{0}} S\left(\vec{x}_{0}\right) e^{-j \vec{k} \cdot \vec{x}_{0}} d^{3} x_{0}
$$

wherer is distance from the scattering volume to the observation location, $P_{0}$ is the magnitude of the incident pressure field and $\vec{K}$,the scattering vector, is defined as the difference between the incident field direction and the scattered field direction, i.e., the direction of observation of a scattered pressure field from the scattering sources[25],

$$
\vec{K}=\vec{k}_{s c}-\vec{k}_{i n c}
$$

Equation (2) can be thought of as the spatial Fourier transform of the object function, $S(\vec{x})$. By observing the scattered field across many different angles, different parts of the object function can be mapped out in terms of the object k-space. For example, forward scattering occurs when the observed scattered field is in the same direction as the incident field yielding 


$$
\vec{K} \approx 0 .
$$

Hence, information in the forward scattering direction relates to low wavenumber values in the $\mathrm{k}$ space of the object, i.e., object features near DC (zero frequency). This in turn suggests the forward scattering direction provides information about object features larger than a wavelength because of the inverse relationship between $k$ and the wavelength. In the backscattered direction, the scattering vector is

$$
\vec{K}=-2 k \hat{k}_{\text {inc }} \text {, }
$$

which suggests that the backscatter provides information abouthigher spatial frequency (wavenumber) components of the scattering object that correspond to smaller scale structures on the order of a wavelength and smaller.

This analysis is not intended to suggest that scatterfrom a solid like concrete with microcracking is best formulated by the Born approximation for p-waves. Rather, the analysis is intended to suggest that the choice of backscatter for analysis would likely be more sensitive to small (sub-wavelength) defects and inclusionsthan the forward scattered field. At ultrasonic frequencies of $50 \mathrm{kHz}$, the wavelength of surface waves in concrete is on the order of $5 \mathrm{~cm}$, whereasmicrocracksin concretetypically are $10 \mu \mathrm{m}$ or smaller in width. Embedded aggregates, pores and other small defects within the cement matrix, as shown in Figure 1, will also contribute to the scattered field. In this study we are particularly interested in the contribution to the scattered field from the microcrack networkin the material while understanding that this contribution superposes on that generated by other natural characteristics of the cement matrix microstructure. Considering this, our interrogations of concrete using $50 \mathrm{kHz}$ ultrasound demonstrate thatthe microcrack scatterers would bevery small relative to a wavelength $(k a<0.001)$ suggesting that a weak scattering model is appropriate for scattering from microcracks. Furthermore, using a weak scatterformulation suggests that the backscatterpower would be proportional to the number density of microcracks, i.e., scatterers, in the field[26],with minimal sensitivity of the backscattered power to the orientation of the cracks with respect to the incident field [27] assuming that other microstructural characteristics of the material are reasonably constant.Hence, the backscattered power can be directly related to the presence and concentration of cracks and, therefore, to degradation processes in concrete.

\section{Methods}

\subsection{Numerical Simulations}

Numerical simulationswereperformed using a commercially availablefinite element analysis (FEA or FE) software (Abaqus/Explicit 6.13) to investigate the influence of distributed microcracking on ultrasonic surface wave signals.A computer having 8 CPUs with $1.60 \mathrm{GHz}$ clock-speed and 16 GB RAM was used for the simulations. The air-coupled ultrasonic testing configuration was simulated using a two-dimensional (2-D) plane strain model, as shown in Figure 2.The 2-D plane strain modelwas utilized instead of the full three-dimensional (3-D)modelto improve the efficiency of numerical computation and the simplicity in physical modeling. This simplification is reasonable for this case because the surface wave responses considered in the experiments correspond to direct wave propagation paths (forward and backward) within one plane. The model comprises air (indicated by blue color in Figure 2), concrete (gray color) and a 
three-layer acoustic barrier(green color), where the material properties were assumed homogeneous and linear-elastic within each. Although concrete is an inhomogeneous and nonlinear material, the material behavior assumptions of the model are reasonable considering that the wavelength of the surface wave at $50 \mathrm{kHz}$ is notablylonger than the largest expected scatterer dimension (ofcoarse aggregate), and that the magnitude of stress field associated with wave propagation is very low. The assumed material properties are summarized in Table 1.The dimensions of the simulated concrete layer are2000 x $500 \mathrm{~mm}$. Two-dimensional plane strain four-node reduced integration elements (CPE4R) were used throughout the model.Reduced time integration,which uses fewer Gaussian coordinates, was employed in the software to minimize the computation time. The mesh size for both the air layer and the acoustic barrier was $1 \mathrm{~mm}$. For the concrete layer, a $1 \mathrm{~mm}$-mesh was used for the near surface region within a depth of $40 \mathrm{~mm}$ from the top surface. The mesh size was gradually increased from $1 \mathrm{~mm}$ to $2.5 \mathrm{~mm}$ as a function of depth within a region from 40 to $80 \mathrm{~mm}$ from the top surface. A2.5 mm mesh was used for the remaining depth region of the concrete layer.The time integration step for the analysis (explicit) was $1 \mu$ s.Note that the time step is well below the critical time step (6 $\mu$ s) needed to accurately represent $50 \mathrm{kHz}$ behavior.

To simulate the dynamics of wave propagation in our experiments, air pressure variation was applied within the air layer near the top surface of the concrete layer assuming a 0.06 MPa maximum magnitude 16-cycle tone burst signal with a center frequency of $50 \mathrm{kHz}$. The dynamic air pressure load was uniformly distributed along the simulated transducer face with a diameter of $30 \mathrm{~mm}$, which simulates the projections of an acoustic wave toward the concrete surface that sets up a forward propagating surface wave that travels to the left.The incident angle of the acoustic wave in air with respect to the surface normal was approximately $9.5^{\circ}$, matching the expected critical angle for surface waves in concrete and air.The resulting dynamic response in the concrete owing to the propagation of surface waveswas monitored at four positions at the top surface of the concrete layer indicated as ACCEL 1, 2, 3 and 4 (vertical surface acceleration) and MEMS 1,2,3 and 4 (acoustic pressure in air $5 \mathrm{~mm}$ above the surface) in Figure 2.Acoustic pressure responses only from the MEMS positions are reported here. These responses simulated leaky surface wave signals detected with air-coupled MEMS sensors in the experimental testing setup. MEMS 1,2 and 3arelocated behind (to the right) the surface point of insonification, thus monitoring backward propagating waves, and MEMS4located ahead of it (to the left), monitoring forward propagating waves. Thethree-layer acoustic barrierindicated by green color in Figure 2simulated the baffle barrier used in the experimental setupto delay and attenuate acoustic waves that propagated backward directly through the air from sender to the air-coupled receiving sensors.

Distributed microcracks in the concrete were simulated by inserting a distinct defect area (40 x $40 \mathrm{~mm})$ bounding thefree surface of the concrete layer that contained a specified amount of volumetric voiding, as shown in Figure 2. To simulate a void, an element mesh in concrete was assigned the properties of air. In this 2-D configuration, each void simulated a single 1-mm wide microcrack oriented perpendicular to the direction of surface wave propagation and running in the outof-plane direction. The size of the voids were much smaller than a wavelength, i.e., approximately 80 times smaller than a wavelength for the P-wave, which suggests that the scattered ultrasound should be insensitive to the shape of the scatterer and could appropriately model the cross sections of microcracks in concrete [28].Void fractions corresponding to 0 
(undamaged), 1, 2, 4 and $8 \%$ of the $40 \times 40 \mathrm{~mm}$ defect area were considered. For each void fraction case, the positions ofthese 1 x 1mm voids (air voids) were randomly assigned within the defect area, and were determined using the random number generating command provided by MATLAB 2014.For each void fraction case, ten independent random spatial distribution patterns of air voids were considered to simulate different surface wave paths along a material having a constant volume of randomly positioned microcracks. The assigned void element sets were automatically applied to the independent FE models using Python software. The effects of relative position of the defect area on the ultrasonic surface wave propagation were evaluated by moving a defect region with constant $2 \%$ void content along nine different locations, indicated by L1 to L9 in Figure 2. The effects of varying severity of microcracking were evaluated by considering a defect region at one location (L6) containingall the considered void fractions.Although the location and severity of the damaged regions were varied in the model, the positions of the air-coupled sender and all receivers and the assumed material properties of the remaining portion of the concrete and the air remained constant throughout the computations.

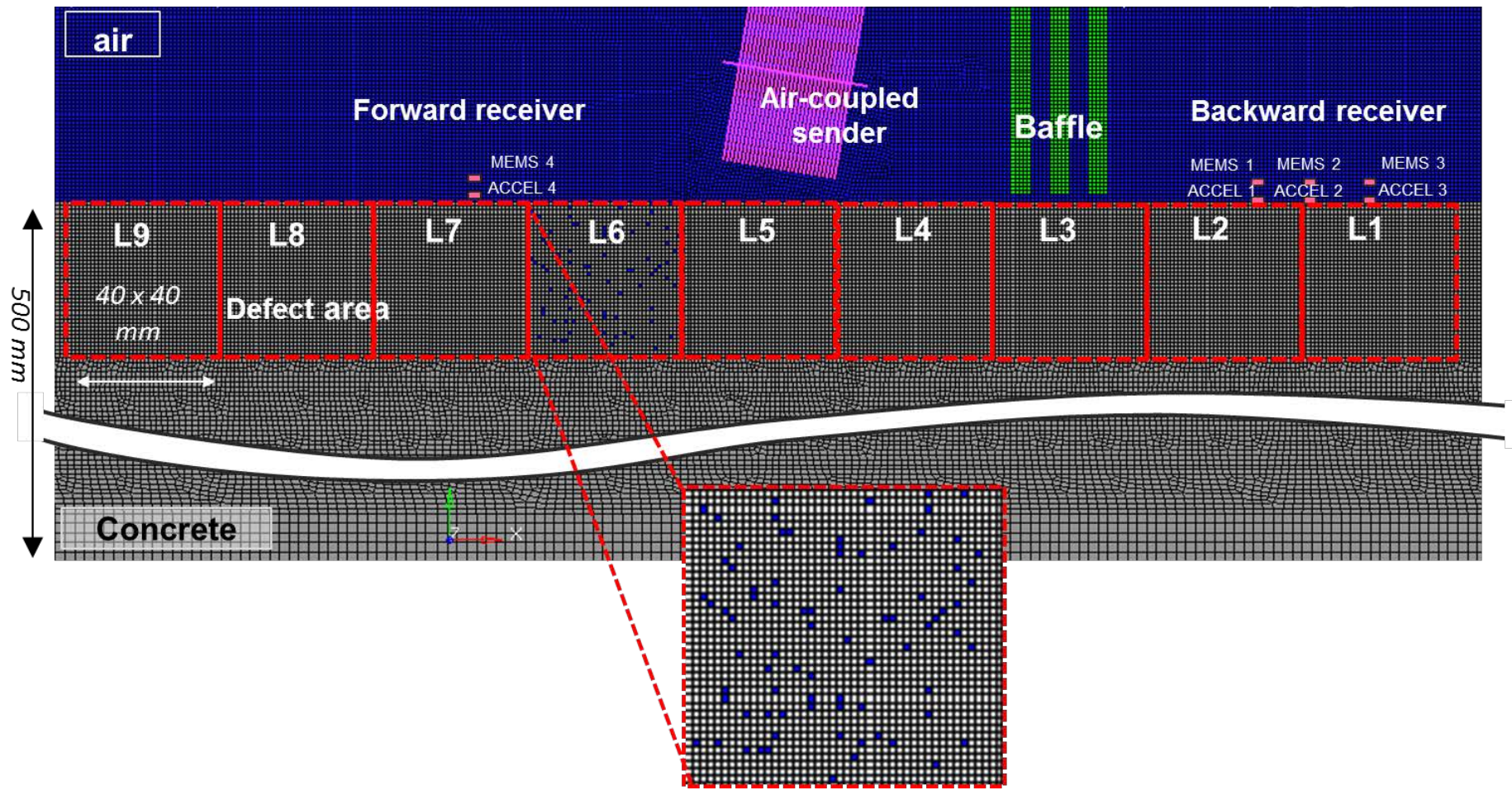

Figure 2 The FE model used to simulate the effects of distributed microcracking in concrete. Configurableregions with simulated microcracking areindicated by red boxes. Individual microcracks simulated by air-filled elements, are indicated by blue color.

Table 1. Summary of material properties for each element.

\begin{tabular}{c|c|c|c}
\hline Elements & Density $\left(\mathrm{kg} / \mathrm{m}^{3}\right)$ & P-wave velocity $(\mathrm{m} / \mathrm{s})$ & Poisson's ratio \\
\hline Air @ 20 $0^{\circ} \mathrm{C}$ & 1 & 343 & 0 \\
Concrete & 2400 & 4058 & 0.2 \\
Baffle & 7800 & 5875 & 0.3 \\
\hline
\end{tabular}




\subsection{Experimental Configuration}

Ultrasonic surface guided waves in concrete were generated and detected using an air-coupled system. The equipment,configuration and concept are shown in Figure 3. This technique is completely contactless as ultrasonicsurface waves are coupled into the concrete from anair-coupled ultrasonic source, andthe resulting propagating and scattered surface waves are coupled back into the air and received by ultrasonic detectors. The equipment consisted of a computer, multi-channel data acquisition system, a modified air-coupled capacitive transmitting transducer[29]and modified aircoupled MEMS receiving transducers[29].Nine MEMS receivers were configured in multi-sensor arrays, with receivers 1 through 7 placed behind the sender, and receivers 8 and 9 ahead of the sender. Although all receivers collected data, only data from receiving sensors 2, 8 and 9 are reported here.

The contactless ultrasonic sender was a capacitive membrane, or electrostatic,transducer (SensComp, Livonia, MI). Capacitance transduction provided a far better match to air (compared with PZT sensors), as the air-pocket /membrane system exhibits much lower acoustic impedance [31]. This transducer module was modified with an external excitation module to control the input signal and improve signal to noise ratio.Silicon-based miniature MEMS acoustic sensors (Knowles, Itasca, IL) were used to detect leaky ultrasonic surface waves emanating from concrete. These sensors were obtained, configured, and evaluated through direct comparison to traditional microphones and capacitive sensors, using a consistent air-coupled ultrasonic source in tests on a concrete sample. The MEMS sensors required a separate power supply (1.5 3 DC volts) and a ceramic capacitor connection $(0.1 \mu \mathrm{F})$, which acts as a high pass filter. TheMEMS sensorshad nominal sensitivity of $7.9 \mathrm{mV} / \mathrm{Pa}$ at $1 \mathrm{kHz}[30]$ with $5 \mathrm{~mm}$ liftoff distance and a working frequency range of 1 to $90 \mathrm{kHz}$.

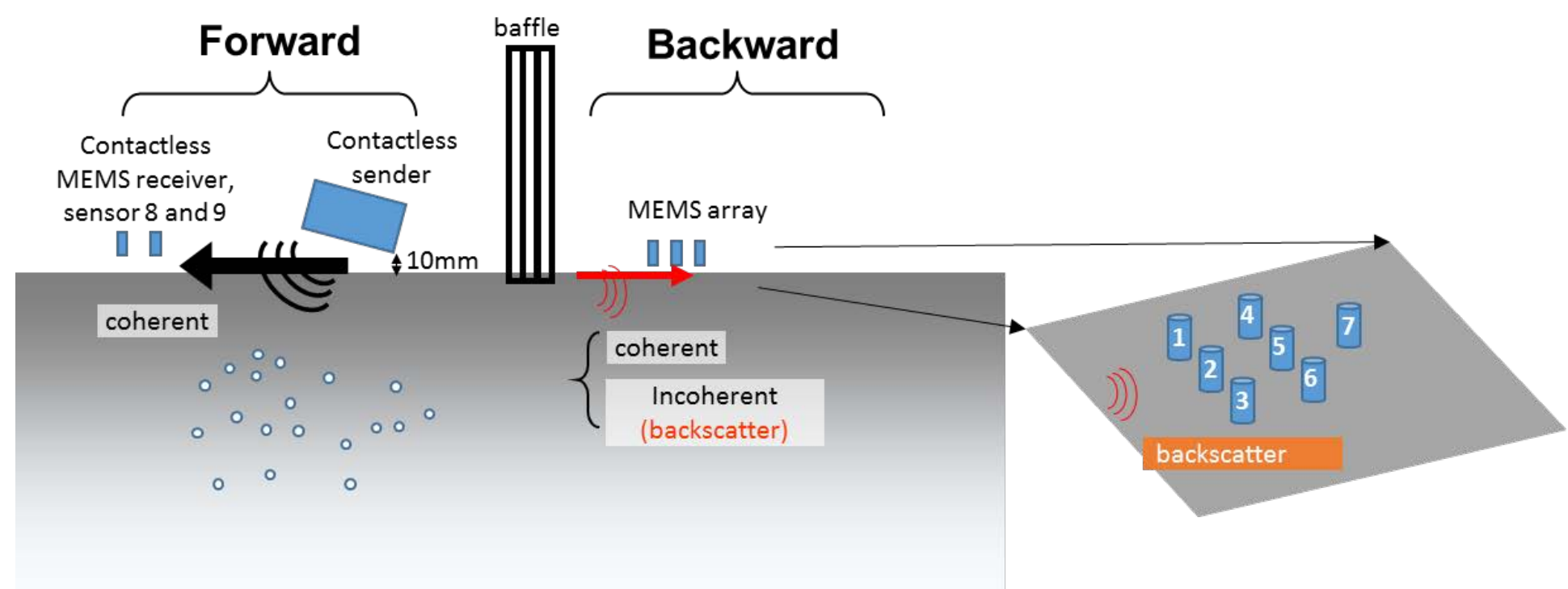

Figure 3Design of fully contactless ultrasonic system to measure backscatter data

A photograph of the bottom side of the ultrasonic testing hardware is provided in Figure 4. A tone burst of 16 cycles with center frequency of $50 \mathrm{kHz}$ was transmitted toward the concrete surface at an angle of approximately $9^{\circ}$ with respect to the concrete surface normal, which matches the expected critical angle for surface waves in concrete and air[29]. As 
shown inFigure 4, the distance between the sender and receiving sensor 8in the forward direction was $80 \mathrm{~mm}$, while the distance between the sender and receiving sensor 2in the backward directionwas $100 \mathrm{~mm}$. Receiving sensors 8 and 9 were separated by $10 \mathrm{~mm}$. Most of the surface energy propagatedin the forward direction. However, some coherent surface wave energy propagated backward because of natural acoustic beam spread from the sending transducers that sends wave energy and also backscatter from the forward propagating wave.For each test case, the whole ultrasonic apparatus was moved along the surface of the sample to acquire ten signals propagating along parallel paths separated by $20 \mathrm{~mm}$. This ensured that each signal came from a different scattering realization from the same crack volume distribution.

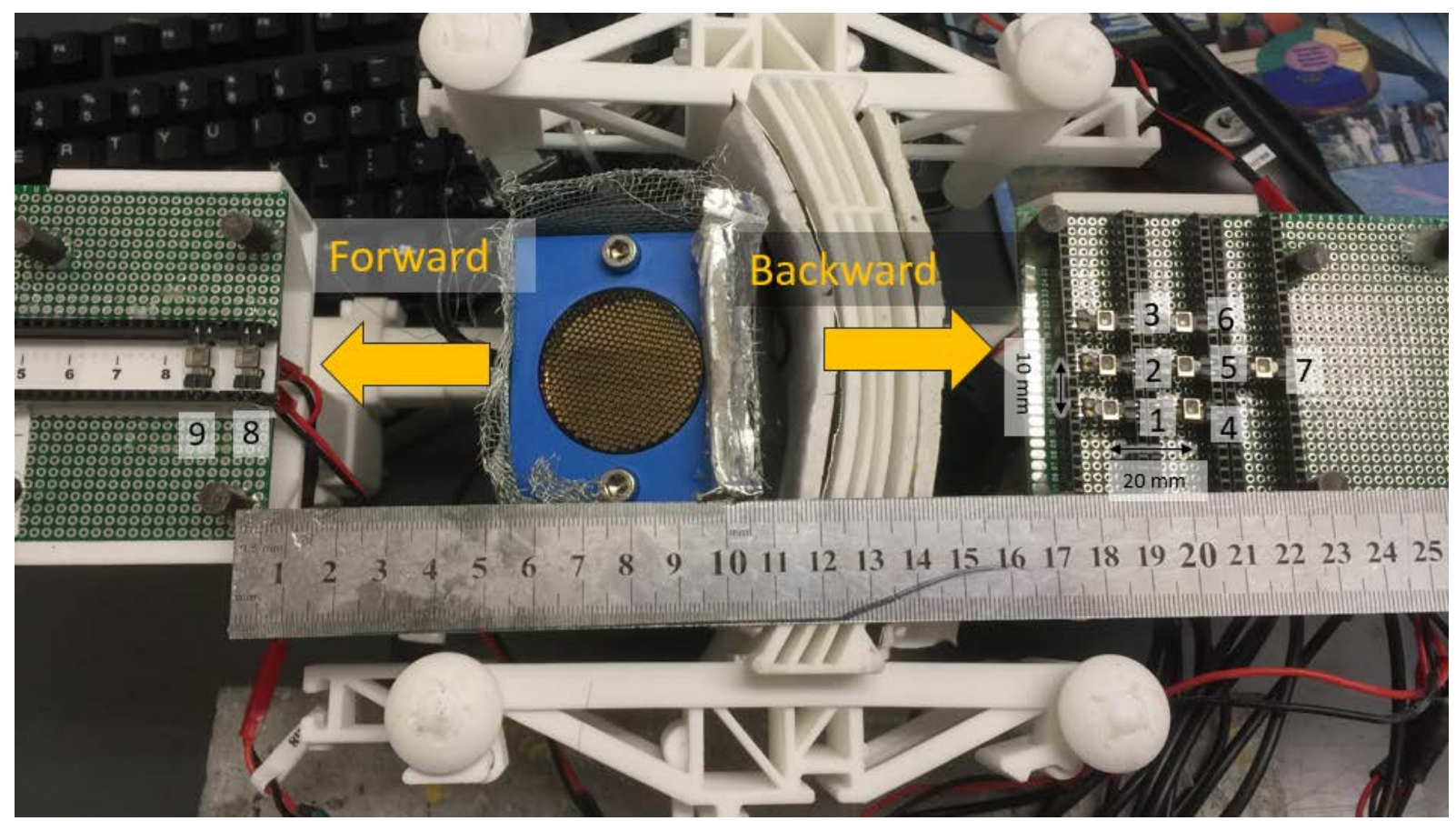

Figure 4Ultrasonic testing hardware showing configuration corresponding to the design in Figure 3

\subsection{Experimental Samples}

Experimental tests were performed in order to characterize different levels (severity) of distributed microcracking in concrete. Sets of experiments were conducted using two different types of samples. The first set of sampleswereconcrete samples with an artificial filler composed of compliant polymer fibers. These fibers are $1 \mathrm{~mm}$ in diameter and50 mm longand are composed of polypropylene. These fiberswere used to simulate uniformly distributed cracking damage (airfilled microcracks and voids) in concrete in a controlled manner. Internally distributed short polymer fibers have been used by researchers to mimic cracking damage in concrete; the fibers simulate discrete internal reflectors with strong scattering characteristics for ultrasonic multiple-scattering studies and can be apportioned in a controlled manner [28, 31]. The level of synthetic damage is defined by the amount of fibers, in terms of percent volume of fiber with respect to total volume, within each sample. The second set of concrete samples contained actual distributed microcracking damage.

In the first set, four different physical specimens were constructed: a 1500 x 1000 x 100 mmPMMA plate and three 300x300x600mm concrete blocks with different polymer fiber contents or filler $(0.0,0.3$, and $0.6 \%$ by volume).Both PMMA and undamagedor no filler $(0.0 \%)$ concrete samples are expected to exhibit low amounts of scattering.The 
PMMA specimen represents a homogeneousmaterial that should provide essentially zero backscattered energy resulting from a forward propagated wave pulse. The $0.0 \%$ fiber concrete naturally contains voidsand mineral aggregate inhomogeneity across a range of length scales that serve as randomly distributed scatterers even in non-damaged samples. All concrete samples had identical age and curing conditions (older than 24 months) and comparable surface conditions. The concrete had a water-to-cement ratio of 0.42 and a maximum aggregate size of $2 \mathrm{~cm}$. The samples had identical constituents, except for the randomly distributed polymer fiber content (soft scatterers): $0.0,0.3$ and $0.6 \%$ by volume.

In the second sample set, a $360 \times 480 \times 140$ mmconcrete slab wascast and then exposed to thermal cycleshock tests to generate real distributed cracking damage in the concrete. The concrete had water to cement ratio of 0.39 and a maximum aggregate size of $2 \mathrm{~cm}$. After casting, the slab specimen was immersed in water for more than 60 daysof curing to continue hydration and minimize drying shrinkage stresses. For the tests, the cured specimen wasimmersed in very warm water for 12 hours then immediately submerged intoice water $\left(1^{\circ} \mathrm{C}\right)$ for a duration of one hour. This entire procedure representsone cycle of thermal cycle process. The thermal cycle processwas repeated six times with a warm water temperature of $68{ }^{\circ} \mathrm{C}$, and then four more times with a warm water temperature of $80^{\circ} \mathrm{C}$. Thus a total of ten thermal cycles were applied. Contactless ultrasonic measurements were applied to the sample after each thermal cycle. Figure 1 shows SEM images of samples extracted from the specimen before and after the thermal cycling was applied. These images verify that our thermal shock treatment generated distributed microcracks within the material.To confirm that significant microcracking damage was created in a progressive fashion with successivethermal cycles, standard vibration resonance tests wereapplied to a separate set of companion concrete samples exposed to the same treatment.Those results are not shown here, but can be found elsewhere [33].A decrease in vibrational mode frequency of the companion specimens with successive thermal cycle applications indicated a monotonic and consistent increase in the level of damage degradation in the concrete.

\subsection{Data Analysis}

The collected signal data were analyzed to extract three test parameters that describe physical phenomena of interest: surface wave forward phasevelocity, surface wave forward signal energy and surface wave backscattered energy. The forward propagating surface wave phasevelocity $V_{s}$ was computed using thearrival of the first positive peak identified inthe forward propagated time signals from sensors 8 and 9 . The phasevelocity, expressed in units of m/s, is defined by

$$
V_{s}=\frac{d 2-d 1}{t 2-\mathrm{t} 1}
$$

where $d 1$ is the position of the first sensor, $d 2$ is theposition of thesecond sensor, $t 1$ is the first peak arrival time from the first sensor, and t2 is the first peak arrival time from thesecond sensor.

The surface wave forward propagated energy was computed by integrating the received time signals in the forward direction from sensor 8over a specified time duration. The average surface wave forward propagated energy, expressed in units of $\mathrm{V}^{2}$, was estimated by averaging the signal energy over signal time ( $\left.t 4-t 3\right)$, 


$$
V I_{j}=\frac{1}{t 4-t 3} \int_{t 3}^{t 4}\left|F v_{j}(t)\right|^{2} d t
$$

where $F v_{j}(\mathrm{t})$ is the $\mathrm{j}$-thtime signal(in units of Volts) corresponding to received surface waves in the forward direction.Figure 5 (top left) shows an example ofa single raw $F v_{j}(\mathrm{t})$ signal. With both the $\mathrm{FE}$ and experimental data, the t4-t3 duration was defined as the time from signal initiation to the third peak arrival time, which is approximately $0.25 \mathrm{~ms}$. This end time was chosen in order to avoid the forward direct acoustic wave in air that arrived at approximately $0.25 \mathrm{~ms}$. To estimate the scattered energy, we developed an energy subtraction analysis (ESA) method. In ESA, tenbackscattered time signals were collected along separate parallel paths on a sampleto form one data setfroma sensor. The ten signals along the individual wave paths were averaged into one signal that approximates the coherent pulse propagating along the test region.The parallel path lines are separated by $20 \mathrm{~mm}$. We selected this separation distance because it is wider thanthe-6- $\mathrm{dB}$ ultrasonic beam width,( 12 mm).In addition, considering the maximum aggregate size of the concrete is 20 $\mathrm{mm}$, a total scanned width of $200 \mathrm{~mm}$ (20 mm x ten scan lines) issufficient to approximate a representative homogeneous response for that region of the material.The scattered energy was computed for waves propagating in the forward (sensor 8) and backward (sensor 2) directions. Significant coherent wave content is expected in the forward propagating signal. But asmallamount of coherent pulse energy was also measured in the backward direction because the beam spread from the sending transducer in air does project some energy in the backward direction. To extract the diffuse scattered field signalB $B_{i}$ from the total wave field that contains coherent pulse content, the averaged signal was subtracted from eachof the 10 individual signals within the set

$$
\mathrm{B}_{\mathrm{i}}=\mathrm{Bv}(\mathrm{t})_{\mathrm{i}}-\frac{1}{N_{\text {path }}} \sum_{i=1}^{N_{\text {path }}} \boldsymbol{B} \boldsymbol{v}(\mathrm{t})_{i}
$$

whereBv $(\mathrm{t})_{\mathrm{i}}$ is $\mathrm{i}^{\text {th }}$ time signal (see Figure 5 top right for an example signal), $\frac{\mathbf{1}}{\boldsymbol{N}_{\text {path }}} \sum_{\boldsymbol{i}=\mathbf{1}}^{\boldsymbol{N}_{\text {path }}} \mathbf{B v}(\boldsymbol{t})_{\mathbf{i}}$ is spatial average over all $B v(t)_{\mathrm{i}}$ defined as $\langle B v(t)\rangle$, and $N_{\text {path }}=10$. Figure 5 (bottom) shows an example of a $\mathrm{B}_{\mathrm{i}}$ signal. The average scattered energy, $\mathrm{EB}_{i}$, expressed in units of $\mathrm{V}^{2}$ for experimental data and $\mathrm{Pa}^{2}$ for $\mathrm{FE}$ simulation data,was obtained by integrating the individual scattered signals over a given period of time

$$
\mathrm{EB}_{\mathrm{i}}=\frac{1}{t 2-t 1} \int_{\mathrm{t} 1}^{\mathrm{t} 2}\left|\mathrm{~B}_{\mathrm{i}}(\mathrm{t})\right|^{2} \mathrm{dt}
$$

The time period,t2-t1,was defined as the duration of 10 cycles $(\sim 0.35 \mathrm{~ms})$, after which the direct acoustic wave in air arrived.

The estimate of $\mathrm{EB}_{\mathrm{i}}$ also depends on the wave energy transduction into the material and the excitation pressure of the transmitter, which is independent of the level of damage in the sample. In order to make the estimate of $\mathrm{EB}_{\mathrm{i}} \mathrm{system}$ independent, the backscattered energy was normalized by the energy in the forward propagating wave, 


$$
E B_{N o r_{-} i}=\frac{E B_{i}}{V I_{i}}
$$

where $V I_{i}$ is the integration of the $i^{\text {th }}$ forward propagated signal. The energy signal of the forward wave also depends on transduction and input pressure amplitude, so these effects are effectively canceled out of the backscattered energy signal.
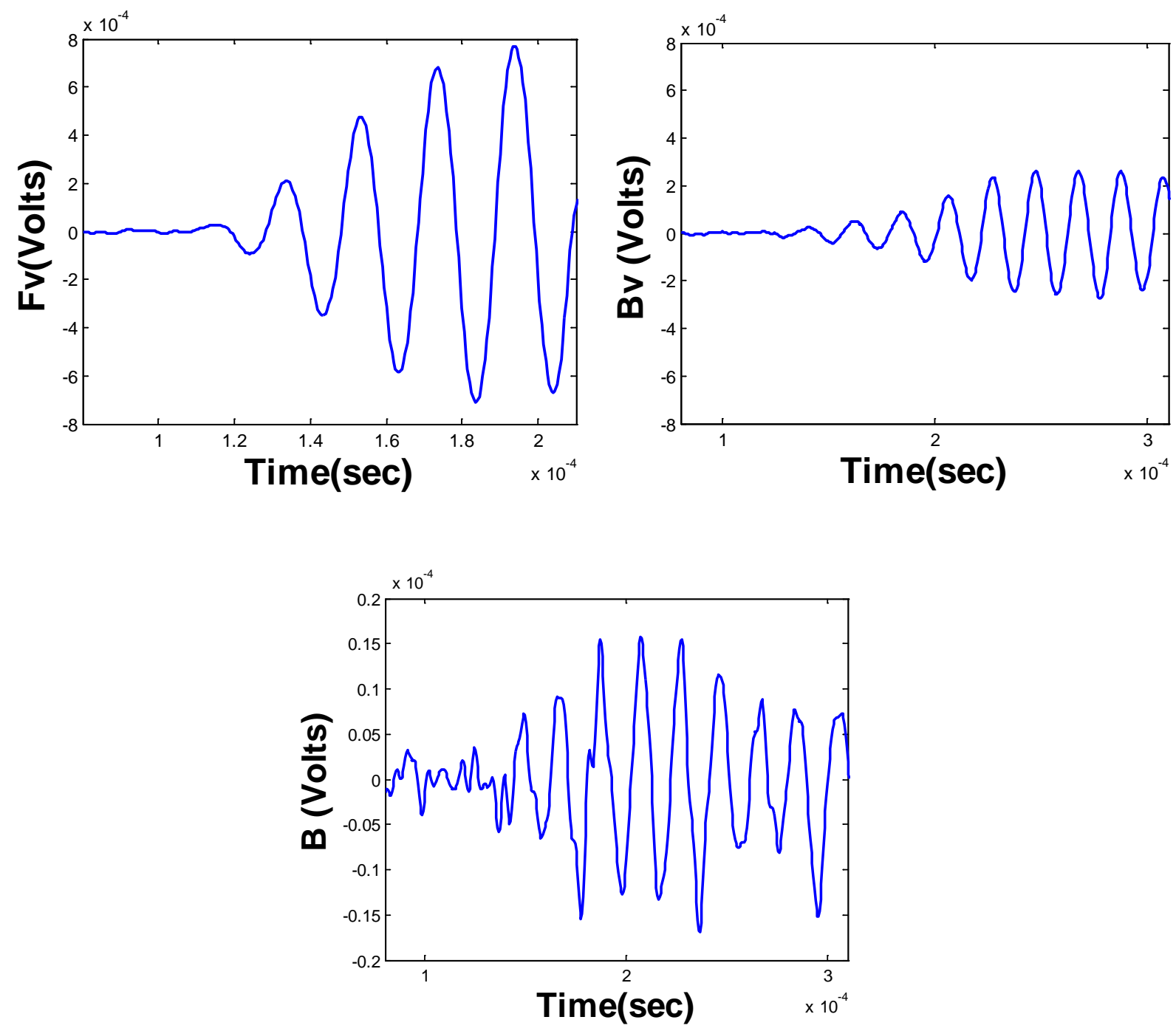

Figure 5Example experimental time-domain data from the specimen containing $0.6 \%$ synthetic damage volume: a single raw forward signal, $F v$ from sensor number 8(top left), a single raw backscattered signal, Bv from sensor 2 (top right), and a single processed time signal, $B_{i}$ calculated by subtractingfrom a single $B v_{i}$ signal the average across the Bv signal set (bottom).

\subsection{Statistical analysis}

Statistical analyses were conducted to quantify the ability ofEB Nor _i $_{1}$ to distinguish between different levels of damage in concrete. Single factor analysis of the variance (ANOVA) was used to test for statistically significant differences (p-values 
$<0.05$ ) between the mean values of $\mathrm{EB}_{\mathrm{Nor}_{\_} \mathrm{i}}$ associated with each damage level (10 estimates ofEB $\mathrm{Nor}_{-} \mathrm{i}$ per damage level). The ANOVA process was calculated with single factor analysis tools in Excel and Minitab(Minitab Inc.,State College,PA).

\section{Results}

\subsection{Simulations}

Figure 6 (left) shows results for wave phasevelocity $\mathrm{V}_{\mathrm{s}}$ (right)and the forward signal energy, $V I_{i}$ obtained from the simulation data.The data set for each damage level is expressed in boxplot format, where the height of the blue box indicates the range of values within which $75 \%$ of the data are contained, the red bar median value, the point mean value and end bars extreme values.Each box plot represents results from ten signals at each different randomly positioned damage level. Wave phasevelocity was observed to decrease with increasing damage level, but not consistently, as shown in Figure 6 (left). The overall reduction in average $V_{S}$ was modest, amounting to less than $2 \%$ drop between minimum and maximum damage levels. The wave phasevelocity at $0 \%$ and $1 \%$ damage levels were not statistically significantly different, although differences among two damage level groups, between $1 \%$ and $2 \%$, and between $4 \%$ and $8 \%$, were statistically significantly different $(\mathrm{p}<0.05)$. The integrated energy in the forward propagating time signals $V I_{i}$, which were obtained from location MEMS 4 indicated inFigure 2,tended to decrease with increasing damage level. $V I_{i}$ decreased with damageup to $4 \%$ but then increased with further damage as shown in Figure 6 (right).The within test data had greater variability compared with $\mathrm{V}_{\mathrm{S}}$ data, as indicated by the height of the data boxes in the plots. Despite the increased withintest variability, statistically significant differences in $V I_{i}$ were observed between damage level groups $0 \%$ and $1 \%, 1 \%$ and $2 \%$, and $2 \%$ and $4 \%$.Statistically significant differences were not observed between the8\% damage level and damage levels $1 \%, 2 \%$ and $4 \%$.
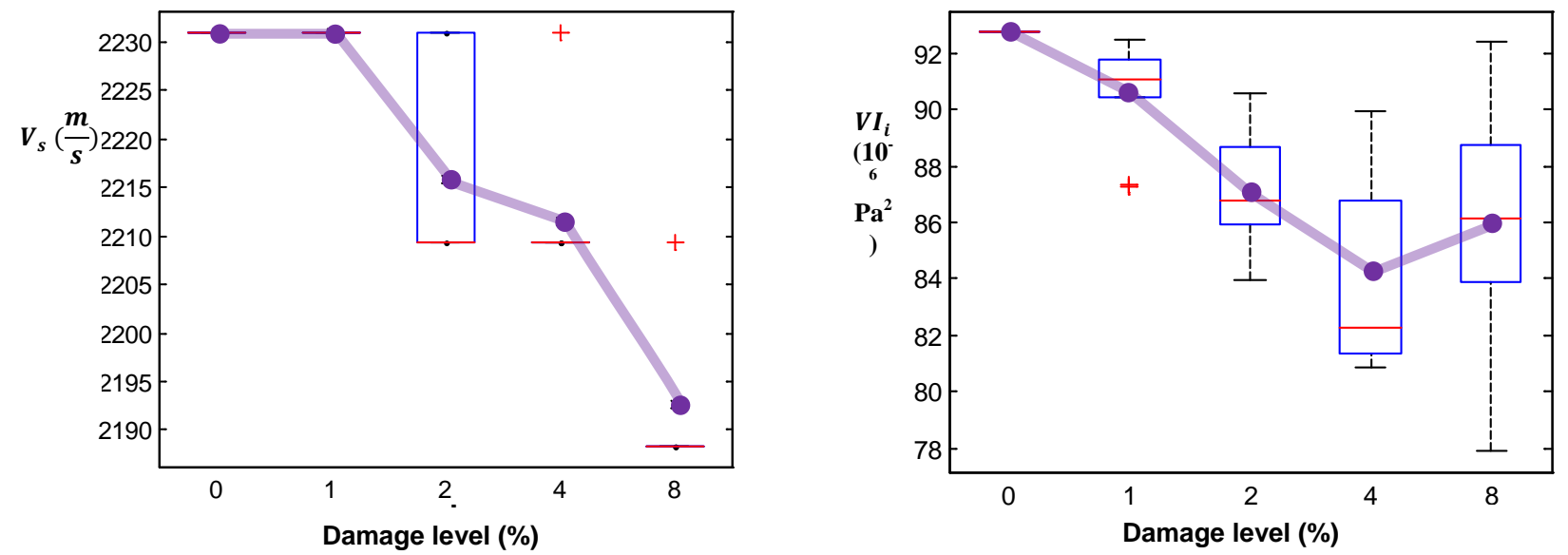

Figure 6Properties of forward-propagating surface waves with different levels of simulated damage: (left) surface wave phasevelocity, and (right) integrated forward-propagating surface wave signal energy, $V I_{i}$; each box plot illustrates data variation for ten signal sets. (FE simulated data)

The computed backscatter energy values $E B_{i}$ are shown inFigure 7 . The $E B_{i}$ data in Figure 7arecollected from the $90 \mathrm{~mm}$ receivingposition (L6 position in Figure 2), which simulated detection with receiving sensor 2in our experimental setup.Although the data do show variability within a given damage level owing to the random positions of the damage, 
distinctions between the damage levels are observed and $E B_{i}$ increases notably and consistently with increasing damage levels. As expected, the undamaged model ( $0 \%$ damage level)exhibited essentially zero backscatter energy, which illustrates the robustness of the data analysis approach to extract a meaningful backscatter energy parameter. Statistically significant differencesin average $\mathrm{EB}_{\mathrm{i}}$ were observed among all damage levels.

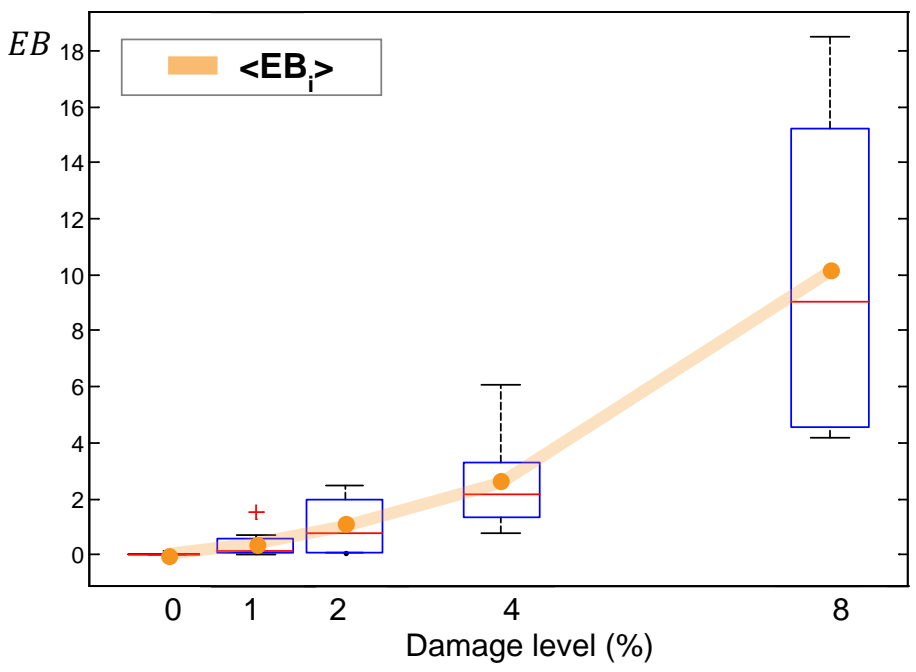

Figure 7Propertiesof averagebackscatter energy parameter $\mathrm{EB}_{i}$ with different levels of simulated damage; box plot illustrates data variation for ten signal sets. (FE simulated data)

Figure 8showsthe plots of the average computed ultrasonic backscatterenergy estimates without (left) and with (right) normalization for two different simulated transmitter output pressures, where the green points and lines indicate double input excitation pressure compared with the blue points and lines. The raw backscattered energy $E B_{i}$ is obtained from Eq. 9, and the normalized backscattered energy $E B_{N_{0}{ }_{-} i}$ from Eq. 10. As input excitation pressure doubles, the resulting $E B_{i}$ also increases proportionally, as shown inFigure 8(left). However, the effects of signal normalization are shown inFigure 8 (right), where $E B_{N_{0 r} i}$ values from two different transmitted pressuresdemonstrate excellent agreement. Thus the normalizing process enabled independent levels of backscatter to be estimated regardless of varyingpressure level of the transmitting transducer.
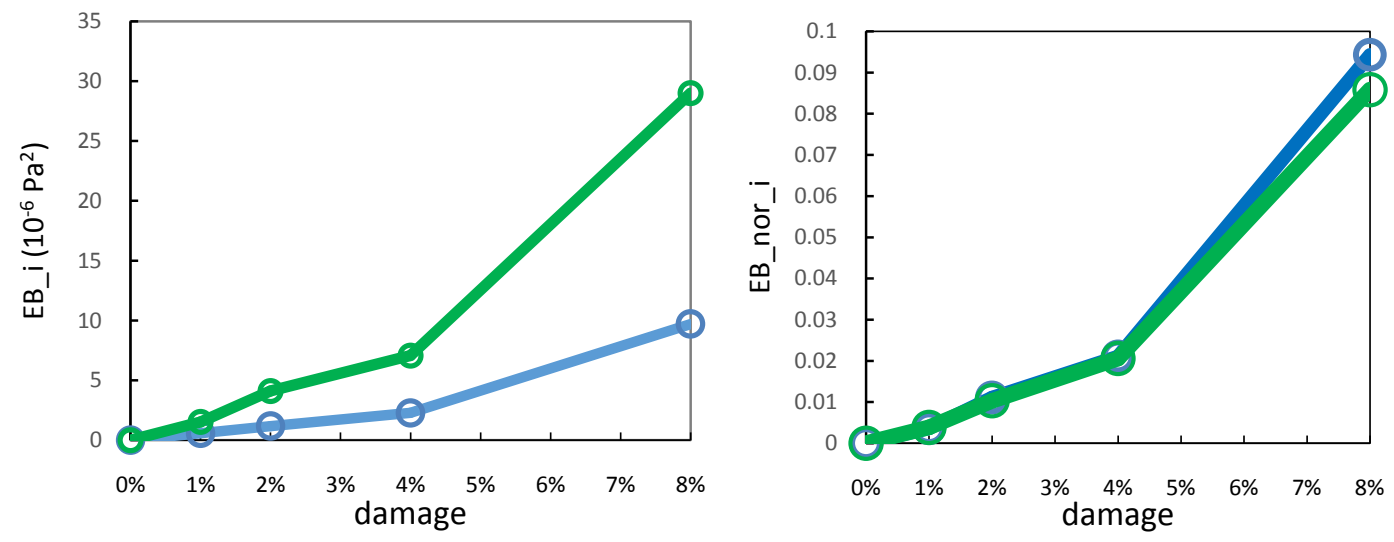

Figure 8Effect of incident wave energy on backscatter parameters for different levels of simulated damage: (left) averages ofbackscattered energy $<E B_{i}>$, (right) average of normalized backscatter energy $<E_{n_{n o r} i}>(F E$ simulated data) 
We also used the FE simulation results to examine the role of incident angle of the transmitting transducer on the estimates of $E B_{i}$. In physical experiments, the configuration of the device may not be consistent across measurements or from one sample to the next. In other words, the angle of incidence of the sending transducer may have small differences from one measurement to the next. Therefore, we examined by simulation the effect of changing the incident angle on the measurements of signal energy in the leaky wave.The optimal incident angle for surface wave excitation can be computed from theory (Snell's law); in the case studied here the expected critical angle for the air-concrete model is $9.5^{\circ}$. Backscatter values were computed using incidence angles between 8 and $12^{\circ}$ in $1^{\circ}$ increments using $\mathrm{FE}$ simulation data forall damage levels. The specific findings are not reported here, but can be found elsewhere [33]. In this casethe incidence angle is reported with respect to the normal direction of the concrete surface. At each angle, ten FE models with different random damage distributions were used to obtain backscatter energy parameters. The results showed that the changes in both parameters, average $\mathrm{EB}_{\mathrm{i}}$ and $E B_{N_{\text {No }} i}$ with respect to incident angle, over the 8 to $12^{\circ}$ incident angle span are modest, with at most a $4 \%$ difference.

In the next set of simulations,the effects of the location of damage with respect to the sensors' position were examined. The simulation results from the three different sensor positions (MEMS 1, MEMS 2, and MEMS 3) showed similar trends. Therefore, the $10 \mathrm{~mm}$ spacing between sensor positions did not appreciably affect backscatter results and here results from only MEMS1 are presented.Referring to Figure 2, L1was the closest to the ultrasonicsender, and L9 was the furthest.Figure 9shows $\mathrm{EB}_{i}$ estimates as a function of damage region position, where ten signals from each configuration were generated by creating 10 different damage profiles for each region. The extracted signal backscatter values, $\mathrm{EB}_{i}$ from locations L1 to L4 are nearly zero. When the damage zone is position underneath or immediately in front the position of the sending transducer, $\mathrm{EB}_{i}$ values rise significantly. These results indicate that the position of the damaged zone affects the results: damage zones that are located behind the sender (i.e. zones L1 to L4) do not affect the backscatter datawhile damage positions directly in front of the sender, e.g. positions L5, L6 and L7, give rise to the largest values of $E B_{i}$; the influence of damage then decreases as itsposition is located farther forward of the sender.Hence, our ESA procedure should allow some degree of damage localization in the material with regard to sensor position. 


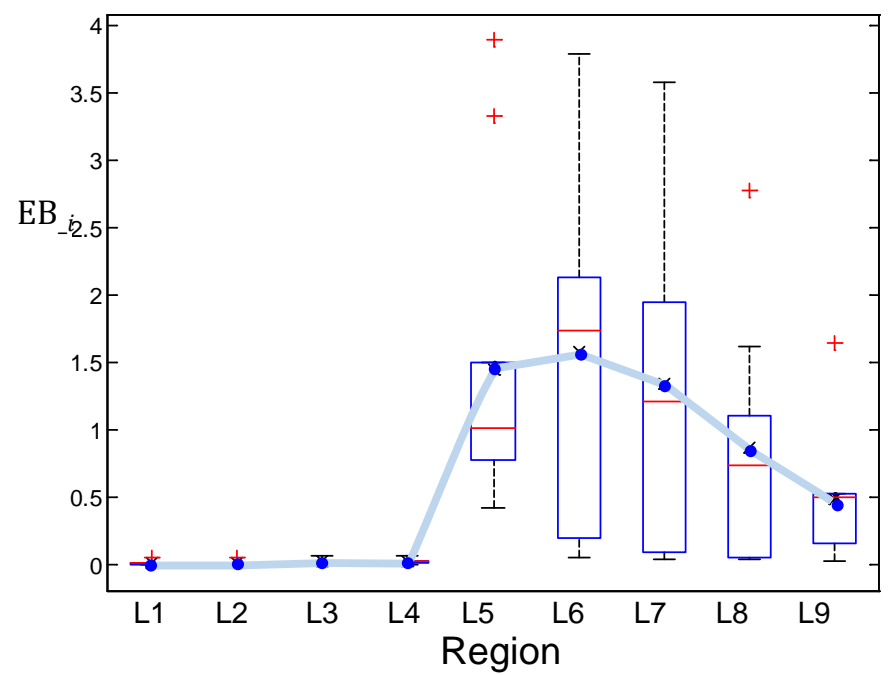

Figure 9Effect of damage positionon normalized backscatter parameter with respect to sensor location; positions of damage zone L1 to L9 illustrated in Figure 2. (FE simulated data)

\subsection{Experiments}

We conducted conventional coherent wave ultrasonic tests byestimatingsurface wave phase velocity $\mathrm{V}_{\text {sand }}$ forward surface wave energy, VI.Data from ten individual parallel scan lines were collected from each sample. Each scan line had22 different sender-receiver positions by moving the positions of the sending and receiving transducers with10 mm steps, from $100 \mathrm{~mm}$ to $320 \mathrm{~mm}$. (top row) shows results of wave phasevelocityestimates,and thebottom rowshows the results ofintegrated forward signal energy, $V I$, from three samples with the different volume percentages of artificial damage. Surface wave phasevelocityprovided no clear distinction among these three damage levels, as shown in (top right). The wavevelocityestimatesbetween the $0.3 \%$ and $0.6 \%$ samples werenot statistically different, although the velocity estimates between the $0.0 \%$ and $0.3 \%$ populations were statistically significant different $(\mathrm{p}<0.05)$.Figure 10 (bottom left) shows the logarithm of the averaged VI from the three samples. No statistically significant differences were observed amongVI estimated for different damage levels.TheVI values exhibitedlarge variations and poor damage sensitivity.
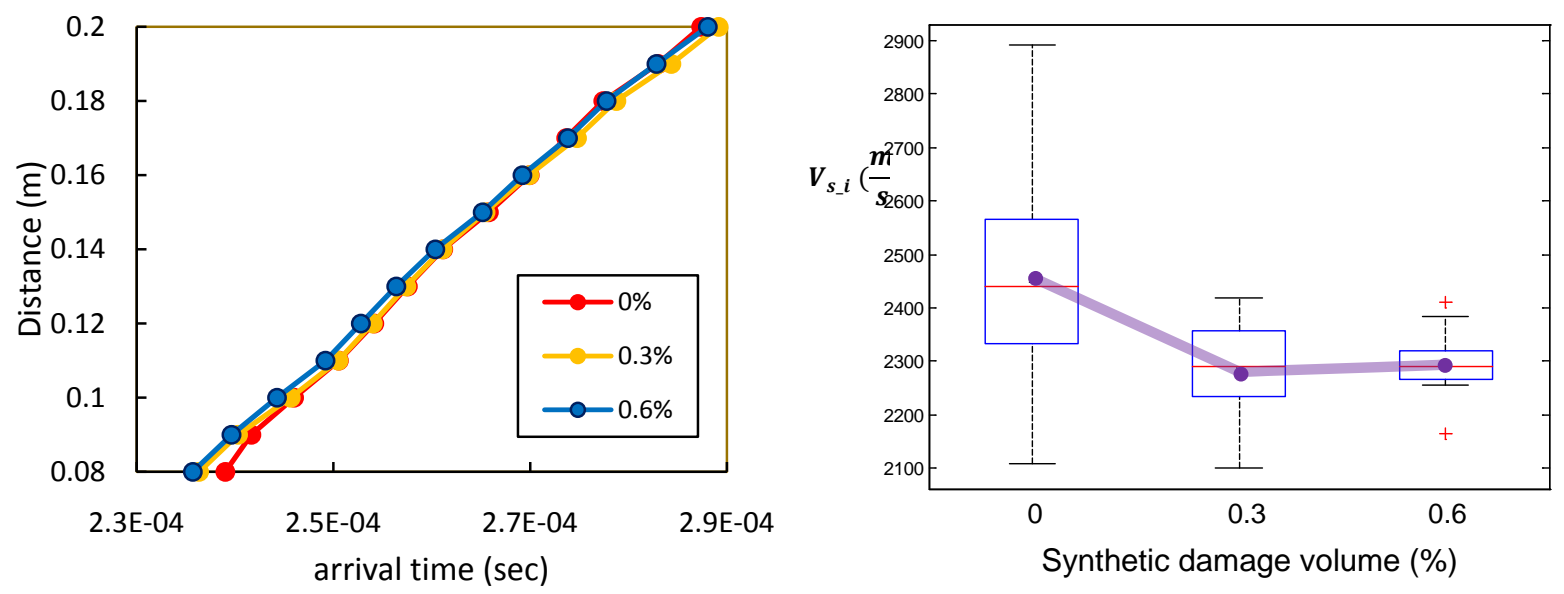

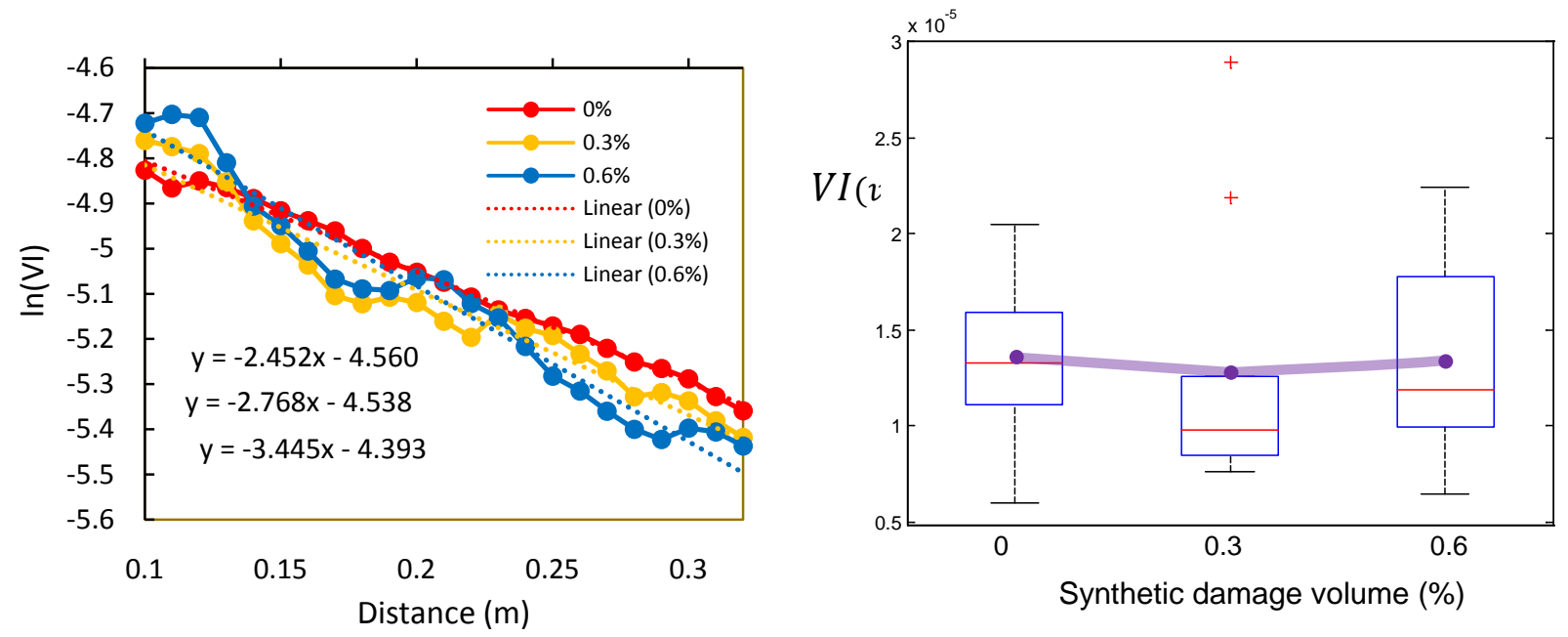

Figure 10Experimental forward propagating ultrasonic data collected from concrete with synthetic damage volume: (top left) typical arrival time profiles, (top right) computed surface wave velocity, (bottom left) typical signal decay curves, and (bottom right) forward propagating wave energyV $I_{i}$.

The developed ESA process was applied to the same data from the fiber concrete samples and also from the PMMA sample. Figure 11shows normalized backscatter energy values, $E B_{n o r_{-} i}$. The trend line for average $E B_{n o r_{-} i}$ (see the purple solid line in the figure) monotonically increased with a higher concentration of fiber filler. Statistically significant differences were observed between all groups. Therefore, $E B_{n o r_{-} i}$ was able to differentiate between the different levels of damage in the concrete samples, and also between PMMA and undamaged concrete.

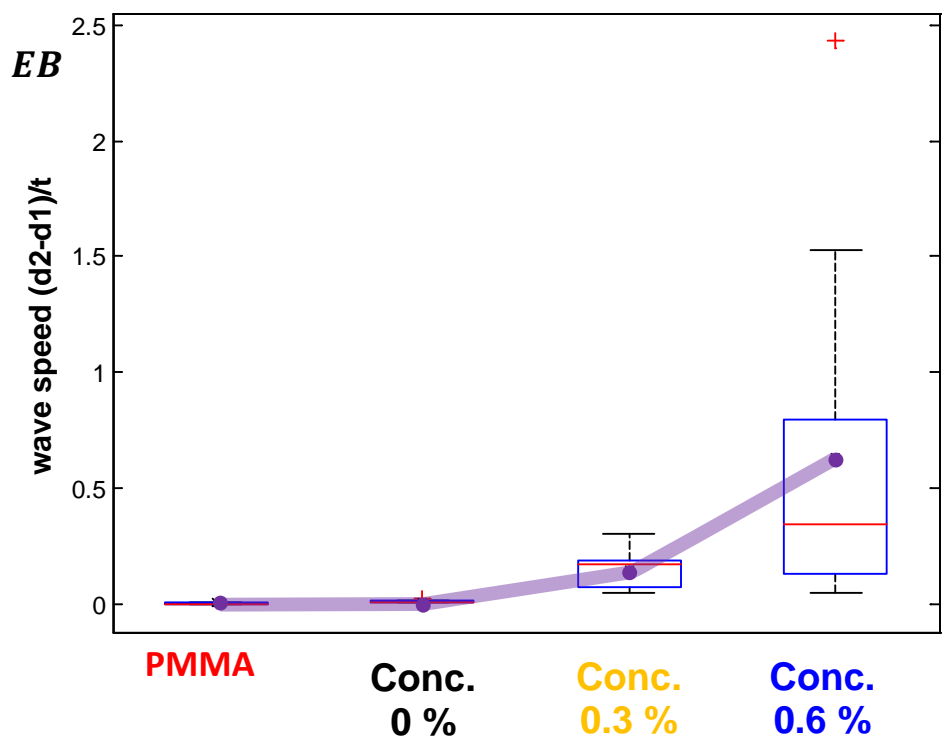

Figure 11Effect of internal fiber filler concentration on normalized backscatter energy.

The same ultrasonic parameters werethenestimated from concrete samples that containedreal distributed microcracking 
contentsubject to the application of ten thermal exposure cycles.Figure 12 shows ultrasonic response data from three different measurement parameters: conventional forward wave speed (Vs), conventional forward pulse energy (VI), and extracted normalized backscatter energy $\left(E B_{n o r_{-} i}\right)$. The conventional ultrasonic parametersVs and VI were not sensitive to changes in distributed microcracking damage as shown in Figure 12 (top right and top left).The forward wave phase velocity and energy estimates provided only a slight decrease with higher levels of damage. The averages of surface wave velocities $\left(\boldsymbol{V}_{\boldsymbol{s}}\right)$ were 2326.8 and 2322.9 for 0 and 10 cycles, respectively.No statistically significant differences were observed between the $\boldsymbol{V}_{\boldsymbol{s}}$ estimated for different damage levels. Pulse energy (VI)also provided no clear distinction and no statistically significant differences between the two damage levels. On the other hand, $E B_{n o r_{-} i}$ exhibited significantly higher values for the damaged state than the nominally undamaged state, with a statistically significant difference ( $\mathrm{p}<$ 0.05 ) in data sets between the two damage levels. Despite the apparently large variance in $E B_{\text {nor }} i$ data observed in both the synthetic fiber and thermal cycle cracking samples, the paper shows statistically relevant (at the 95\% confidence level) evidence that the tests results among different levels of cracking volume are distinct. This statistical analysis supports the viability of the method for in situ application.
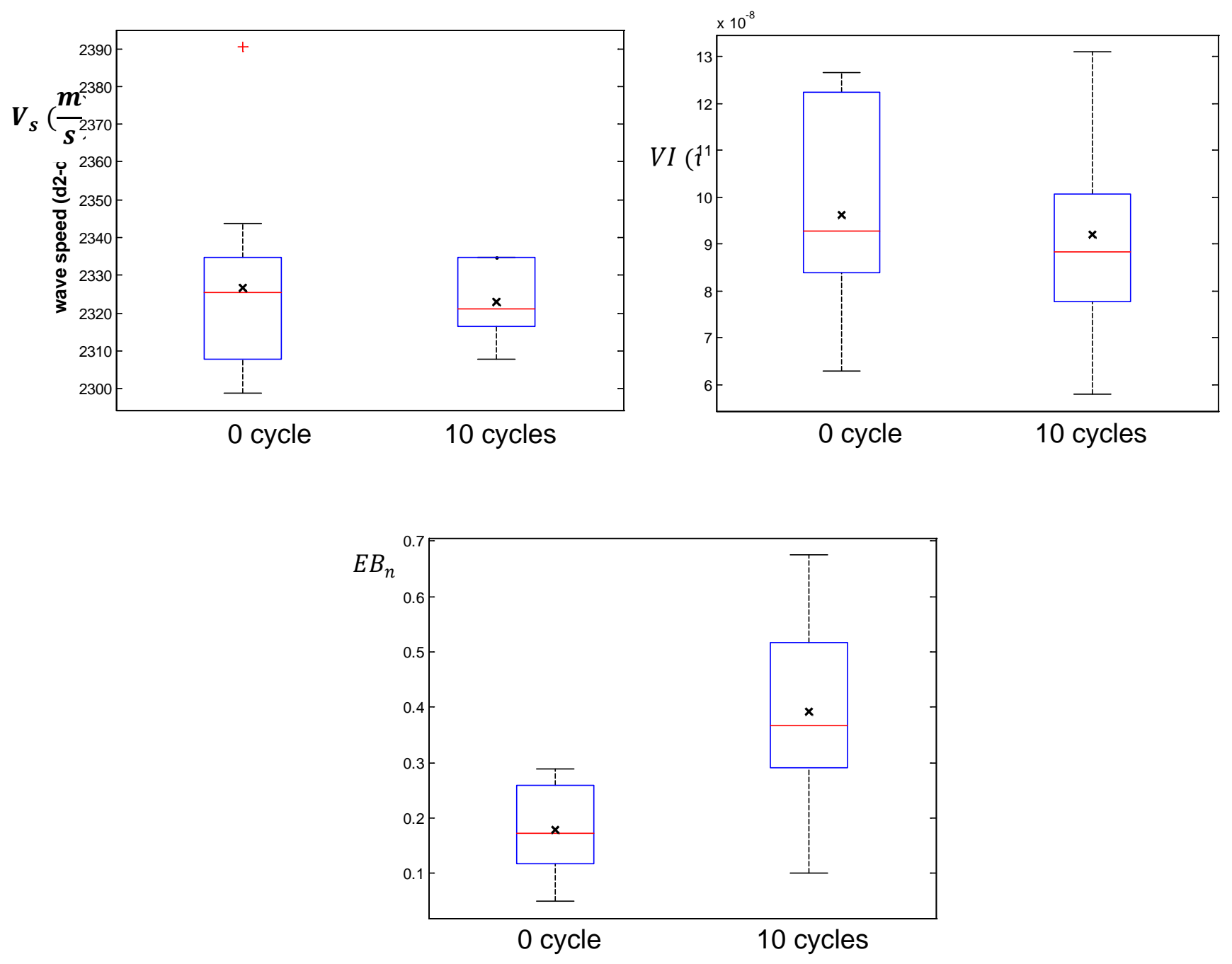

Figure 12. Ultrasonicdata from concrete sample with distributed thermal cracking: (top left) surface wave (Vs), (top right) pulse energy, VI, (bottom) normalized backscatter energy, EB $B_{n o r_{-} i}$. 


\section{Discussion}

Both simulation and experimental data demonstratedthat contactless ultrasonic interrogation could quantify the damage from distributed microcracking in concrete samples. The technique relied on generating a surface wave in the concrete using a source coupled through air and calculating the diffuse backscattered energy in the propagating surface wave. Sensors close to the surface were able to detect a superposition of a coherent forward propagating wave and a backscattered wave coupled from the concrete back into the air. The forward wave could be effectivelysubtracted out leaving the backscattered wave field.

The 2-D simulations were not meant to serve as direct simulation of the experiments carried out, but rather were conducted to provide a prediction of the trends that would accompany scattering in the forward and backward directions from small cracks compared to wavelength. While not perfect, the 2-D simulations provided important insights into the physics of the underlying problem. Therefore, direct comparison ofthe backscattered energy values from simulation results (e.g. Figure 6) with experimental results (e.g. Figure 10) versus level of damageis not warranted. The value in conducting the 2-D simulation results was twofold: 1) to quantify the importance of backscatter versus forward scatter with regard to sensitivity to microcrack damage, and 2) to assess the importance of changes in experimental configuration to consistent estimation of the backscattered energy. Both experimental and simulation results demonstratedthe same trendsversus level of damage. The backscatter results from both simulations and experiments had much higher sensitivityto crack damage compared to conventional methods examining forward propagating waves. Furthermore, the simulations provided additional evidence that deployment of the device in the field would be robust because small changes in the experimental configuration, which can occur through multiple deployments, would not detract from the ability to consistently estimate microcrack damage.

Simulations predicted that the backscattered energy was dependent on the pressure output of the source. In order to mitigate this dependence, the backscattered energy was divided by the energy quantified in the forward direction. By inserting this normalization step, the technique was system independent in the sense that different transmitter outputs, and also receiver sensitivities, would not provide statistically significant differences in the results. Furthermore, the importance of the incidence angles was explored and found that within the range of 8 to $12^{\circ}$ the backscattered energy estimates were relatively flat. Therefore, small differences in the incidence angle are not expected to produce widely varying estimates of $\mathrm{EB}_{i}$ and thus the incident angle of the transmitter does not need to be precisely controlled in order to get acceptable and consistent backscatter results. Finally, changes in the position of the receivers behind the transmitter did not result in large changes in the estimated backscattered energy. Additional simulation studies examined the importance of the backscatter versus the forward scatter in characterizing microcracking damage.The results indicate that the field scattered in thebackward direction was highly sensitive to the amount of microcracking damage in the sample; on the other hand, the signal in the forward direction was not very sensitive to the microcracking damage.The results of FE simulation provided better understanding of the backscatteranalysis. Backscatter energy, $E B_{\text {nor }_{-} i}$, more than doubled with an increase from 4 to $8 \%$ of the void fraction (damage level). Forward coherent approaches (e.g., wave velocity) were more limited in their abilities to detect and quantify microcracking damage. In the case of the forward wave phase velocity a monotonically changing velocity was not observed versus damage.Therefore, the forward wave phase velocity 
was not able to uniquely characterize the damage level.

The experimental data from air-coupled sensors demonstrated sufficient signal-to-noise ratio to provideconsistent and high quality data. Physical testing results inconcrete sampleswith artificial filler (Figure 11)demonstrated a monotonic increase in the backscatter energy, $E B_{\text {nor }_{-} i}$, although the energy was smaller than predicted by the FE results possibly because of the amplitude of the excitation difference. However, similartrends were observed in the numerical simulations. The concordance between the results predicted from simulations and results from physical experiments provide additional confidence that contactless ultrasonic backscatter estimation can provide quantification of distributed microcracking damage.

\section{Conclusions}

The following conclusions are drawn based on the results and discussion presented in the paper. First,the technique was able to extract high quality scattered field data using a fully contactless ultrasonic system. Parameterization of thenormalized backscattered data allowed characterization of the extent of distributed microcrackingdamage within the material with much higher sensitivity than forward propagating coherent pulse analysis (i.e., forward wave phase velocity and forward energy estimates).Second,the normalized backscatter energy, $E B_{\text {nor } \_} i$, parametercould be estimatedessentially independent of the testing setup. Third, the simulations suggested that most of the backscattered response arosefrom distributed cracks located in front of the ultrasonic sender, while cracks behind the sender had little contribution to the backscattered energy. Finally, the normalized backscatter energiesincreasedmonotonicallywith increases inthe internal microcracking damage content.Therefore, our results indicate that characterization of sub-wavelength sized defects is possible with non-contact ultrasonic surface wave backscatter field analysis. The proposed method offers a sensitive, contactless and practical NDT approach to monitor actual distributed microcracking in concrete.

\section{Acknowledgments}

The work reported in this paper was carried out under support from the National Science Foundation, through grant CMS1300546.

\section{Cited references}

1. Geoffrey Baskir et. al., “2013 report card,” ASCE, 2013. [Online]. Available: http://www.infrastructurereportcard.org/a/\#p/state-facts/sources.

2. D. J. Naus, “A compilation of elevated of elevated temperature concrete material property data and information for use in assessments of nuclear power plant reinforced concrete structures," ORNL/TM2009/175, Oak Ridge National Laboratory, Oak Ridge, Tennessee, 2009.

3. American Concrete Institute (ACI) Committee 228, "Nondestructive Test Methods for Evaluation of Concrete in Structures,” Rep. No.ACI 228.2R-98, Farmington Hills, Mich., Farmington Hills, Mich., 1998.

4. S. F. Selleck, E. N. Landis, M. L. Peterson, S. P. Shah, and J. D. Achenbach, "Ultrasonic investigation of concrete with distributed damage,” ACI Mater. J., vol. 95, no. February, pp. 27-36, 1998. 
5. S. Ould Naffa, M. Goueygou, B. Piwakowski, and F. Buyle-Bodin, "Detection of chemical damage in concrete using ultrasound.,” Ultrasonics, vol. 40, no. 1-8, pp. 247-51, May 2002.

6. $\quad$ E. N. Landis and S. P. Shah, “Frequency-dependent stress wave attenuation in cement-based materials,” J. Eng. Mech., vol. 121, no. June, pp. 737-743, 1995.

7. W. Punurai, J. Jarzynski, J. Qu, J.-Y. Kim, L. J. Jacobs, and K. E. Kurtis, “Characterization of multi-scale porosity in cement paste by advanced ultrasonic techniques,” Cem. Concr. Res., vol. 37, no. 1, pp. 38-46, Jan. 2007.

8. F. Saint-Pierre, P. Rivard, and G. Ballivy, "Measurement of alkali-silica reaction progression by ultrasonic waves attenuation,” Cem. Concr. Res., vol. 37, no. 6, pp. 948-956, Jun. 2007.

9. P. A. Gaydecki, F. M. Burdekin, W. Damaj, D. G. John, and P. A. Payne, "The propagation and attenuation of medium-frequency ultrasonic waves in concrete : a signal analytical approach," Meas. Sci. Technol., vol. 3, pp. 126-134, 1992.

10. W. Suaris and V. Fernando, "Ultrasonic Pulse Attenuation as a Measure of Damage Growth during Cyclic Loading of Concrete,” ACI Mater. J., no. 84, pp. 185-193, 1988.

11. D. G. Aggelis, E. Z. Kordatos, M. Strantza, D. V. Soulioti, and T. E. Matikas, "NDT approach for characterization of subsurface cracks in concrete,” Constr. Build. Mater., vol. 25, no. 7, pp. 3089-3097, Jul. 2011.

12. J. Gross, J.-Y. Kim, L. J. Jacobs, K. E. Kurtis, and J. Qu, “Evaluation of near surface material degradation in concrete using nonlinear Rayleigh surface waves,” in AIP Conference Proceedings, 2013, vol. 1309, pp. 1309-1316.

13. X. J. Chen, J.-Y. Kim, K. E. Kurtis, J. Qu, C. W. Shen, and L. J. Jacobs, "Characterization of progressive microcracking in Portland cement mortar using nonlinear ultrasonics,” NDT E Int., vol. 41, no. 2, pp. 112-118, Mar. 2008.

14. J. Chen, A. R. Jayapalan, J.-Y. Kim, K. E. Kurtis, and L. J. Jacobs, "Rapid evaluation of alkali-silica reactivity of aggregates using a nonlinear resonance spectroscopy technique,” Cem. Concr. Res., vol. 40, no. 6, pp. 914-923, Jun. 2010.

15. A. A. Shah and Y. Ribakov, "Non-linear ultrasonic evaluation of damaged concrete based on higher order harmonic generation,” Mater. Des., vol. 30, no. 10, pp. 4095-4102, Dec. 2009.

16. G. Kim, C.-W. In, J.-Y. Kim, K. E. Kurtis, and L. J. Jacobs, “Air-coupled detection of nonlinear Rayleigh surface waves in concrete-Application to microcracking detection,” NDT E Int., vol. 67, pp. 64-70, 2014.

17. P. Shokouhi, "Monitoring of Progressive Microcracking in Concrete Using Diffuse Ultrasound," in 6th European Workshop on Structural Health Monitorin, 2012, pp. 1-8.

18. E. Larose and S. Hall, "Monitoring stress related velocity variation in concrete with a $2 \times 10(-5)$ relative resolution using diffuse ultrasound.,” J. Acoust. Soc. Am., vol. 125, no. 4, pp. 1853-6, Apr. 2009.

19. D. P. Schurr, J. Y. Kim, K. G. Sabra, and L. J. Jacobs, "Damage detection in concrete using coda wave interferometry,” NDT E Int., vol. 44, no. 8, pp. 728-735, 2011.

20. P. Anugonda, J. S. Wiehn, and J. a. Turner, “Diffusion of ultrasound in concrete,” Ultrasonics, vol. 39, no. 6, pp. 429-35, Oct. 2001.

21. F. Deroo, L. J. Jacobs, J.-Y. Kim, J. Qu, and K. Sabra, "Damage detection in concrete using diffuse ultrasound measurements,” in AIP Conference Proceedings, 2010, vol. 1509, no. 2010, pp. 1509-1516.

22. A. Quiviger, C. Payan, J.-F. Chaix, V. Garnier, and J. Salin, "Effect of the presence and size of a real macro-crack on diffuse ultrasound in concrete,” NDT E Int., vol. 45, no. 1, pp. 128-132, Jan. 2012.

23. F. Deroo, "Damage detection in concrete using diffuse ultrasound measurements and an effective 
medium theory for wave propagation in multi-phase materials,” MS Thesis, Georgia Institute of Technology, 2009.

24. R. E. Green, “Non-contact ultrasonic techniques.,” Ultrasonics, vol. 42, no. 1-9, pp. 9-16, Apr. 2004.

25. P. M. Morse and K. U. Ingard, Theoretical Acoustics, Ch 8: The Scattering of Sound. New Jersey: Princeton University Press, 1939.

26. K. A. Wear, "Measurement of dependence of backscatter coefficient from cylinders on frequency and diameter using focused transducers--with applications in trabecular bone.," J. Acoust. Soc. Am., vol. 115, no. 1, pp. 66-72, 2004.

27. K. A. Wear and G. R. Harris, "Frequency dependence of backscatter from thin, oblique, finite-length cylinders measured with a focused transducer-with applications in cancellous bone," J Acoust Soc Am, vol. 124, no. 5, pp. 3309-3314, 2008.

28. D. R. Palmer, "Rayleigh scattering from nonspherical particles,” J. Acoust. Soc. Am., vol. 99, no. 4, pp. 1901-1912, 1996.

29. S. Ham and J. S. Popovics, "Contactless ultrasonic scanning approach toward automated inspection of concrete structures,” Autom. Constr., 2015.

30. S. Ham and J. S. Popovics, "Application of Micro-Electro-Mechanical Sensors Contactless NDT of Concrete Structures,” Sensors, vol. 15, no. 4, pp. 9078-9096, 2015.

31. D. W. Schindel, D. A. Hutchins, and M. Sayer, "The design and characterization of micromachined aircoupled capacitance transducers," IEEE Trans. Ultrason. Ferroelectr. Freq. Control, vol. 42, no. 1, pp. 42-50, Jan. 1995.

32. D. G. Aggelis and T. Shiotani, "Effect of Inhomogeneity Parameters on Wave Propagation in Cementitious Material,” ACI Mater. J., vol. April, no. 105, pp. 187-193, 2009.

33. S. Ham, "Damage Assessment in Reinforced Concrete using Contactless ultrasound," Ph.D. Thesis, University of Illinois at Urbana-Champaign, 2015. 